\title{
Improving Receiver Performance of Diffusive Molecular Communication with Enzymes
}

\author{
Adam Noel, Student Member, IEEE, Karen C. Cheung, and Robert Schober, Fellow, IEEE
}

\begin{abstract}
This paper studies the mitigation of intersymbol interference in a diffusive molecular communication system using enzymes that freely diffuse in the propagation environment. The enzymes form reaction intermediates with information molecules and then degrade them so that they cannot interfere with future transmissions. A lower bound expression on the expected number of molecules measured at the receiver is derived. A simple binary receiver detection scheme is proposed where the number of observed molecules is sampled at the time when the maximum number of molecules is expected. Insight is also provided into the selection of an appropriate bit interval. The expected bit error probability is derived as a function of the current and all previously transmitted bits. Simulation results show the accuracy of the bit error probability expression and the improvement in communication performance by having active enzymes present.
\end{abstract}

Index Terms-Molecular communication, intersymbol interference, diffusion, nanonetwork

\section{INTRODUCTION}

Recent interest in the design of nanonetworks, where communicating devices have functional components that are on the order of nanometers in size, has emerged for applications in areas such as biomedicine, environmental monitoring, and manufacturing; see [2], [3]. The devices themselves could share information over potentially longer distances, on the micrometer scale and further. This communication capability is essential if the entire devices are very small since they would have limited individual processing capacity. Molecular communication is a nanonetwork design strategy where a transmitter emits information molecules that are carried to an intended receiver. It is a bio-inspired approach that can take advantage of the many mechanisms in cells and subcellular structures that already use the emission of molecules for communication. By utilizing biological components, such as genetically modified cells, we might hope to design networks that are inherently biocompatible for implementation inside of living organisms.

The simplest propagation method in molecular communication is free diffusion, which can be modeled as a random walk. Molecules that are released by a transmitter can freely

Manuscript received January 22, 2013; revised October 16, 2013; accepted December 4, 2013. This work was presented in part at the 7 th International Conference on Bio-Inspired Models of Network, Information, and Computing Systems (BIONETICS 2012) in Lugano, Switzerland [1]. This work was supported by the Natural Sciences and Engineering Research Council of Canada, and a Walter C. Sumner Memorial Fellowship. Computing resources were provided by WestGrid and Compute/Calcul Canada.

The authors are with the Department of Electrical and Computer Engineering, University of British Columbia, Vancouver, BC, Canada, V6T $1 Z 4$ (email: \{adamn, kcheung, rschober\}@ece.ubc.ca). R. Schober is also with the Institute for Digital Communication, Friedrich-Alexander-Universität Erlangen-Nürnberg (FAU), Erlangen, Germany (email: schober@lnt.de). diffuse away without any external energy or infrastructure requirements. Diffusion can be very fast over short distances, and is a common means of communication in nature; many cellular processes rely on diffusion for limited quantities of molecules to efficiently propagate both within and between cells, as described in [4, Ch. 16]. Many researchers have also adopted diffusion for the design of molecular communication networks, cf. e.g. [5]-[23].

The average distance travelled by a diffusing molecule is proportional to the square root of the time that it takes to diffuse. So, molecular communication systems have to deal with increasingly longer propagation times as the receiver is placed further away. The general lack of control over where molecules diffuse means that a large number of molecules is required to ensure that a sufficient number arrive at the receiver instead of diffusing away. Furthermore, the receiver's ability to differentiate between the arrival of the same type of molecule emitted at different times is reduced by how long it takes for those molecules to leave the proximity of the transmitter and receiver. Unless there is a mechanism in place to remove excess information molecules from the environment, the transmission rate between a single transmitter and receiver is limited by the on-going proximity of previously emitted molecules, i.e., intersymbol interference (ISI).

The current literature on diffusion-based molecular communication has primarily dealt with ISI via passive strategies where the transmitter must wait sufficiently long for previously-emitted information molecules to diffuse away before it can release more molecules, thereby limiting the maximum transmission rate. For example, ISI has often been ignored, as in [5]-[9], [17], or it has been assumed that interfering molecules are released no earlier than the previous bit interval, as in [10]-[13], [18], [19]. ISI from all previous transmissions has been considered in 1-dimensional diffusion environments in [20], [21] and in 2-dimensional environments in [22], where the Viterbi algorithm is applied at the receiver to optimally detect emissions from a transmitter that uses molecule shift keying (though the Viterbi algorithm may be too complex for practical implementation in small bio-inspired devices). An upper-bound on the capacity of a 3-dimensional diffusive environment, accounting for all ISI, was recently derived in [23].

Communications capacity can be significantly improved by adding a mechanism that actively transforms information molecules so that they are no longer recognized by the receiver. In general, chemical reactant molecules could perform this role, but then they must be provided in stoichiometric excess relative to the information molecules, otherwise their 


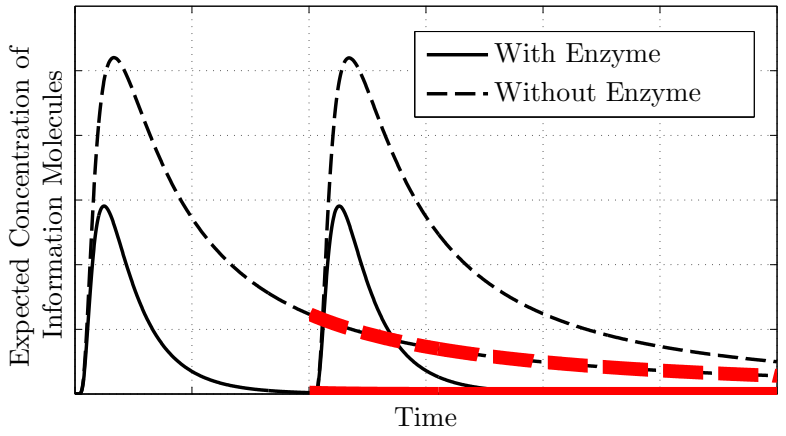

Fig. 1. A sample comparison of the expected concentration of information molecules at a receiver with and without enzymes present in the propagation environment. In each case, the transmitter emits two impulses of molecules. The relative quantity of ISI from the first impulse, shown as a thicker red line, is much greater without active enzyme molecules.

capacity to transform the information molecules will degrade over time. Catalysts, on the other hand, lower the activation energy for biochemical reactions but do not appear in the stoichiometric expression of the complete transformative reaction; unlike reactants, catalysts are not consumed. Specifically, enzymes are catalytic biomolecules that can have the advantage of very high selectivity for their substrates; see [4, Ch. 16]. Some enzymes are already used in nature for the purpose of reducing ISI; for example, acetylcholinesterase is an enzyme in the neuromuscular junction that hydrolyzes acetylcholine as it diffuses to its destination, as described in detail in [24, Ch. 12]. Furthermore, another mechanism regenerates acetylcholine at the transmitter from its hydrolyzed components so that it can be re-used in future emissions.

We are interested in using enzymes in the propagation environment due to their selectivity and because a single enzyme can be recycled to react many times. No additional complexity at either the transmitter or receiver is required. The reduction in ISI would enable transmitters to release molecules more often, simultaneously increasing the data rate and decreasing the probability of erroneous transmission. There would also be less interference from neighbouring communication links, so independent transmitter-receiver pairs could be placed closer together than in an environment dominated by diffusion alone.

The potential for information molecules to participate in a chemical reaction mechanism has usually been considered only at the receiver, as in [9], [14], [25]. Papers that have considered information molecules reacting in the propagation environment include [15], [16], [26]. In [15], the spontaneous destruction and duplication of information molecules are treated as noise sources but were not deliberately imposed to improve communication. In [16], the exponential decay of information molecules was considered via simulation as a method to reduce ISI. However, information was measured as the total number of molecules to reach the receiver, so the achievable information rate actually decreased when information molecules were allowed to decay. The placement of enzymes along the boundaries of the propagation environment with the goal of reducing ISI was proposed in [26] but analytical results were not provided.

In this paper, we present a model for analyzing diffusion- based molecular communication systems when there are enzymes present throughout the entire propagation environment. The enzymes react with the information molecules via Michaelis-Menten kinetics, which is a common mechanism for enzymatic reactions; see [27, Ch. 10]. We first introduced this scenario in [1], where we showed that enzymes reduce the "tail" created when we rely on diffusion alone. In Fig. 1] we present a visual example of the degradation of the diffusion "tail" when enzymes are present. It is clear that, although enzymes reduce the expected peak concentration, significantly less ISI can be expected due to the on-going degradation of information molecules throughout the propagation environment. This paper expands the work presented in [1] and makes the following contributions:

1) As in [1], we present a lower bound expression on the expected number of information molecules observed at a receiver due to a transmitter that uses binary modulation to emit impulses of molecules when enzymes are present in the propagation environment.

2) We derive the time at which the maximum number of information molecules is expected, both with and without enzymes. We also derive an upper bound expression on the time for the expected number of molecules to decrease from the maximum number to an arbitrary fraction of the maximum. This analytically shows that, for a given level of ISI, a shorter bit interval can be achieved by adding enzymes, and provides insight into the selection of an appropriate bit interval.

3) We design a simple detector where the receiver counts the number of molecules observed at the instant when the number of molecules is expected to be maximum and the observed number of molecules is compared to a binary decision threshold.

4) We derive the bit error rate of this scheme for the first emission by the transmitter and then as a function of the current and all previous emissions. This derivation is the first to consider all ISI in evaluating the bit error probability of a receiver in a 3-dimensional diffusive environment.

5) As in [1], we justify a particle-based simulation framework and describe its implementation in our reactiondiffusion system.

The rest of this paper is organized as follows. In Section III we introduce our system model for a single transmission link using binary modulation, including the degradation of information molecules via Michaelis-Menten kinetics. A lower bound expression on the expected number of observed molecules at the receiver when enzymes are present is derived in Section III and compared with the baseline, no-enzyme scenario. The performance analysis of the receiver, where we calculate the signal degradation time and derive the bit error rate for the simple detection scheme, is presented in Section IV. In Section $\mathrm{V}$, we describe the simulation framework and provide some insight into the selection of appropriate parameter values. In Section VI, we present and discuss numerical and simulation results. Conclusions and the on-going direction of our research are described in Section VII 


\section{Physical Model}

There is a transmitter fixed at the origin of an unbounded 3-dimensional fluidic environment. The receiver is a fixed spherical observer with radius $\left|\vec{r}_{o b}\right|$ and volume $V_{o b}$. It is centered at location $\left\{x_{0}, y_{0}, z_{0}\right\}$ where $\vec{r}_{0}$ is the vector from the origin to $\left\{x_{0}, y_{0}, z_{0}\right\}$. The receiver is a passive observer; molecules can diffuse through it as they do through the entire environment.

There are three diffusive molecular species in the system that we are interested in: $A$ molecules, $E$ molecules, and $E A$ molecules. $A$ molecules are the information molecules that are released by the transmitter. These molecules have a natural degradation rate that is negligible over the time scale of interest, but they are able to act as substrates with enzyme $E$ molecules. We apply Michaelis-Menten kinetics, which is generally accepted as the fundamental mechanism for enzymatic reactions (see [24], [27]), to the $A$ and $E$ molecules:

$$
E+A \underset{k_{-1}}{\stackrel{k_{1}}{\rightleftharpoons}} E A \stackrel{k_{2}}{\longrightarrow} E+A_{P}
$$

where $E A$ is the intermediate formed by the binding of an $A$ molecule to an enzyme molecule and $A_{P}$ is the degraded (product) $A$ molecule. The reaction rate constants for the reactions as shown are $k_{1}$ in molecule ${ }^{-1} \mathrm{~m}^{3} \mathrm{~s}^{-1}, k_{-1}$ in $\mathrm{s}^{-1}$, and $k_{2}$ in $\mathrm{s}^{-1}$. We see that $A$ molecules are irreversibly degraded by the reaction defined by $k_{2}$ while the enzymes are released intact so that they can participate in future reactions. $A_{P}$ molecules are ignored once they are formed because they cannot participate in future reactions and they are not recognized by the receiver (we do not consider the re-generation of $A$ molecules from $A_{P}$ molecules at the transmitter). Throughout this paper, we refer to the three reactions in (11) associated with $k_{1}, k_{-1}$, and $k_{2}$ as the binding, unbinding, and degradation reactions, respectively.

We use a common notation to refer to parameters of each molecular species. We define these parameters for arbitrary species $S$, which could be either $A, E$, or $E A$ molecules. The number of molecules of species $S$ is given by $N_{S}$, and its concentration at the point defined by vector $\vec{r}$ and at time $t$ in molecule $\cdot \mathrm{m}^{-3}$ is $C_{S}(\vec{r}, t)$. For compactness, we will generally write $C_{S}(\vec{r}, t)=C_{S}$. We assume that every molecule of each species $S$ diffuses independently of all other molecules. We assume that all free molecules are spherical in shape so that we can state that each molecule diffuses with diffusion constant $D_{S}$, found using the Einstein relation as [24, Eq. 4.16]

$$
D_{S}=\frac{k_{B} T}{6 \pi \eta R_{S}},
$$

where $k_{B}$ is the Boltzmann constant $\left(k_{B}=1.38 \times 10^{-23} \mathrm{~J} / \mathrm{K}\right)$, $T$ is the temperature in kelvin, $\eta$ is the viscosity of the medium in which the molecules are diffusing $\left(\eta \approx 10^{-3} \mathrm{~kg} \cdot \mathrm{m}^{-1} \mathrm{~s}^{-1}\right.$ for water at $25^{\circ} \mathrm{C}$ ), and $R_{S}$ is the molecule radius. Thus, the units for $D_{S}$ are $\mathrm{m}^{2} / \mathrm{s}$. The diffusion of a single molecule along one dimension has variance $2 D_{S} t$, where $t$ is the diffusing time in seconds [24, Eq. 4.6].

Communication occurs as follows. The transmitter emits impulses of $A$ molecules, where the number of molecules emitted is $N_{A e m}$. This is a common emission scheme in

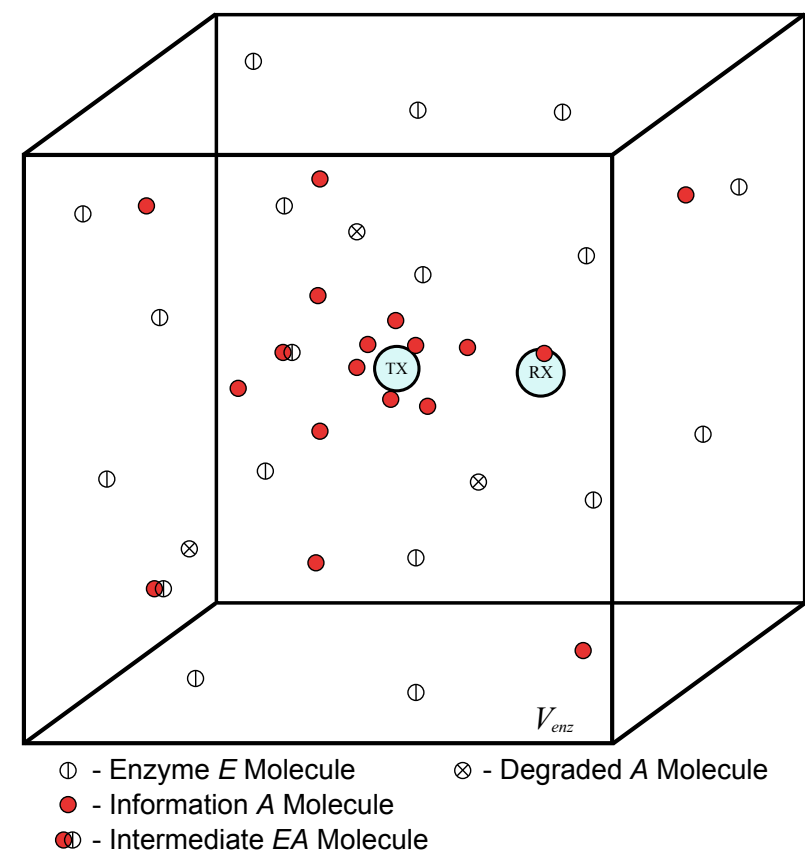

Fig. 2. The bounded space $V_{\text {en }}$ showing a uniform distribution of enzyme $E$ molecules (enzymes are shown as circles with vertical lines through them). $V_{\text {enz }}$ inhibits the passage of $E$ so that the total concentration of free and bound $E$ remains constant. Information $A$ molecules (shown as red circles) are emitted by the transmitter and can diffuse beyond $V_{\text {enz }}$. Intermediate $E A$ molecules can form when an $A$ molecule binds to an $E$ molecule. When an intermediate dissociates, it can leave the $A$ molecule degraded (shown as a circle with an $\mathrm{X}$ through it).

the molecular communication literature; see, for example, [8], [14], [28], [29]. We deploy binary modulation with constant bit interval $T_{B}$, where $N_{\text {Aem }}$ molecules are released at the start of the interval for binary 1 and no molecules are released for binary 0 . This method is also known as ON/OFF keying. $N_{E}$ enzyme $E$ molecules are randomly (uniformly) distributed throughout a finite cubic volume $V_{\text {enz }}$ that includes both the transmitter (TX) and receiver (RX), as shown in Fig. 2 with the transmitter at the center. The diffusion of the $E$ molecules is restricted to within $V_{e n z}$, such that the total concentration of the free and bound enzyme in $V_{e n z}, C_{E_{T o t}}$, is constant and equal to $N_{E} / V_{e n z}$ (the local concentration of free and bound enzyme in any subregion of $V_{e n z}$ does vary over time). Further details on the implementation of a finite $V_{e n z}$ in the simulation framework are provided in Section $\mathrm{V}$, but we assume in our analysis that $V_{e n z}$ is infinite in size.

The receiver counts the number of free (unbound) $A$ molecules that are within the receiver volume, without disturbing those molecules. For a practical bio-inspired system, the $A$ molecules would need to bind to receptors on either the receiver surface or within the receiver's volume, but we assume perfect passive counting in order to focus on the propagation environment.

\section{ObSERVATIONS AT THE RECEIVER}

Generally, the spatiotemporal behavior of the information, enzyme, and intermediate molecules can be described using a system of reaction-diffusion partial differential equations. Even though these equations are deterministic, they will enable 
stochastic simulation. In this section, we use the reactiondiffusion partial differential equations to derive the expected number of information molecules at the receiver.

\section{A. Diffusion Only}

Fick's Second Law describes the motion of arbitrary $S$ molecules undergoing independent diffusion as [24, Ch.4]

$$
\frac{\partial C_{S}}{\partial t}=D_{S} \nabla^{2} C_{S}
$$

for species $S$ where $D_{S}$ is the diffusion coefficient of the species. Closed-form analytical solutions for partial differential equations are not always possible and depend on the boundary conditions that are imposed. For comparison, we first consider the scenario of no enzyme present, i.e., $N_{E}=0$. So, we immediately have $C_{E}=C_{E A}=0 \forall \vec{r}$, $t$, and we only consider the diffusion of $A$ molecules. The expected scaled (by $N_{\text {Aem }}$ ) impulse response at a distance $|\vec{r}|$ from the transmitter is then [24, Eq. 4.28]

$$
C_{A}=\frac{N_{A e m}}{\left(4 \pi D_{A} t\right)^{3 / 2}} \exp \left(\frac{-|\vec{r}|^{2}}{4 D_{A} t}\right),
$$

where $t$ is the time since the $N_{\text {Aem }}$ information molecules were released. Eq. (4) is the form that is typically used in molecular communication to describe the local concentration at the receiver (where $\vec{r}=\vec{r}_{0}$, the vector from the transmitter to the center of the receiver); the receiver is assumed to be a point observer, as in [30], [31], or the concentration throughout the receiver volume is assumed to be uniform and equal to that expected in the center, as in [14]. Eq. (4) is the baseline against which we evaluate our proposed system design.

\section{B. Reaction-Diffusion}

We now include active enzymes in our analysis. The general reaction-diffusion equation for species $S$ is [32, Eq. 8.12.1]

$$
\frac{\partial C_{S}}{\partial t}=D_{S} \nabla^{2} C_{S}+f\left(C_{S}, \vec{r}, t\right),
$$

where $f(\cdot)$ is the reaction term. Applying the principles of chemical kinetics (see [27, Ch. 9]) to Michaelis-Menten kinetics in (1), we write the complete set of reaction-diffusion equations for the species in our environment as

$$
\begin{aligned}
\frac{\partial C_{A}}{\partial t} & =D_{A} \nabla^{2} C_{A}-k_{1} C_{A} C_{E}+k_{-1} C_{E A}, \\
\frac{\partial C_{E}}{\partial t} & =D_{E} \nabla^{2} C_{E}-k_{1} C_{A} C_{E}+k_{-1} C_{E A}+k_{2} C_{E A}, \\
\frac{\partial C_{E A}}{\partial t} & =D_{A} \nabla^{2} C_{E A}+k_{1} C_{A} C_{E}-k_{-1} C_{E A}-k_{2} C_{E A} .
\end{aligned}
$$

This system of equations is highly coupled due to the reaction terms and has no closed-form analytical solution under our boundary conditions; we must make some simplifying assumptions:

1) We assume that the degradation reaction is relatively very fast, i.e., $k_{2} \rightarrow \infty$.

2) We assume that the unbinding reaction is relatively very slow, i.e., $k_{-1} \rightarrow 0$.
From the first assumption, we can claim that $C_{E}$ remains close to the total concentration of free and bound enzyme, i.e., $C_{E_{\text {Tot }}}=N_{E} / V_{\text {enz }}$, over all time and space, since there will never be a significant quantity of bound enzyme. Thus, $C_{E A}$ remains small over all time and space. Before applying explicit bounds on $C_{E}$ and $C_{E A}$, it is sufficient for a solution to assume that they are both steady and uniform (i.e., they are constant) and it can then be shown that, in our system, (6) has solution

$$
C_{A} \approx \frac{N_{A e m}}{\left(4 \pi D_{A} t\right)^{3 / 2}} \exp \left(-k_{1} C_{E} t-\frac{|\vec{r}|^{2}}{4 D_{A} t}\right)+k_{-1} C_{E A} t,
$$

and we ignore (7) and (8). Next, we apply the upper bound on $C_{E}$ (i.e., $C_{E_{T o t}}$ ) and use the second assumption to apply a lower bound on $k_{-1} C_{E A}$ (i.e., 0) to write the lower bound on the expected impulse response as

$$
C_{A} \geq \frac{N_{A e m}}{\left(4 \pi D_{A} t\right)^{3 / 2}} \exp \left(-k_{1} C_{E_{T o t}} t-\frac{|\vec{r}|^{2}}{4 D_{A} t}\right),
$$

which is intuitively a lower bound because the actual degradation due to enzymes can be no more than if all enzymes were always unbound. The tightness of this lower bound depends directly on the accuracy of our two assumptions about the reaction rates. The actual expected concentration will be between (10) and the diffusion-only case (4), but will become closer to (10) if the two assumptions are more accurate (i.e., if the assumptions are not accurate, then the mitigation of ISI by adding enzymes is less than expected). In general, this lower bound loses accuracy as $E A$ is initially created $\left(C_{E}<C_{E_{T o t}}\right)$, but it eventually improves with time for non-zero reaction rates as all $A$ molecules are degraded and none remain to bind with the enzymes $\left(C_{A}, C_{E A} \rightarrow 0, C_{E} \rightarrow C_{E_{T o t}}\right.$, as $\left.t \rightarrow \infty\right)$.

An alternate solution for (6) can be derived without our two assumptions about the reaction rate constants $k_{-1}$ and $k_{2}$, where it is only assumed that $C_{E A}$ is constant. This is a common step in the analysis of Michaelis-Menton kinetics; see [27, Ch. 10] and its use when considering enzymes only at the receiver in [9]. The resulting expression is similar to [10], where the binding rate $k_{1}$ is replaced with $k_{1} k_{2} /\left(k_{-1}+k_{2}\right)$, but it is an approximation and not a lower bound.

Eq. (10) can be directly compared with (4). The presence of enzyme molecules results in an additional decaying exponential term. This decaying exponential is what will eliminate the "tail" that is observed under diffusion alone. An immediate result from 10 is that increasing either the binding rate $k_{1}$ or the total enzyme concentration $C_{E_{T o t}}$ will result in a fasterdecaying "tail" and thus decrease ISI, albeit at the cost of also decreasing the useful signal in the desired bit interval.

We will assume throughout the remainder of this paper that the expected concentration of information $A$ molecules within the receiver is uniform and equal to that expected at the center of the receiver. We studied the accuracy of this assumption in [33], where we showed it is accurate for a receiver that is sufficiently far from the transmitter. We have already established that the receiver is able to count the number of free $A$ molecules that are within the receiver volume, so we can readily convert the expected concentration into the expected number of observed $A$ molecules at the receiver, 
$\overline{N_{A o b}}(t)$. Using 10 with enzymes and 4 without enzymes we can write

$$
\overline{N_{A o b}}(t)=C_{A}\left(\vec{r}_{0}, t\right) V_{o b},
$$

for the expected number of observed molecules, where $\vec{r}_{0}$ is the vector from the origin to the center of the receiver.

\section{ReCEIVER PERformance AnAlysis}

In this section, we first consider the signal degradation with enzymes and provide a method to calculate an appropriate bit interval $T_{B}$. Then, we derive the bit error rate of the considered receiver and provide approximations that facilitate closed-form expressions. We design the reception mechanism such that the receiver counts the number of free $A$ molecules observed within the receiver volume $V_{o b}$ at the instant when the expected number of molecules is maximal (assuming that the transmitter emits molecules at the start of the bit interval). A single decision threshold is used for the receiver to determine whether a binary 1 or binary 0 was sent by the transmitter. This is a relatively simple reception mechanism that facilitates analysis and approximates a physically realizable scheme. For example, if a biochemical response mechanism were triggered at the receiver when the information molecule concentration reached a threshold level, then the threshold is most likely to be exceeded when the maximum number of information molecules is expected. The more realistic case, where the threshold could be exceeded at any time, is an interesting problem that we leave for future work.

\section{A. Signal Degradation with Enzymes}

From 10 and $(11)$, the number of information $A$ molecules expected at the receiver is

$$
\overline{N_{A o b}}(t) \geq \frac{V_{o b} N_{A e m}}{\left(4 \pi D_{A} t\right)^{3 / 2}} \exp \left(-k_{1} C_{E_{T o t}} t-\frac{\left|\vec{r}_{0}\right|^{2}}{4 D_{A} t}\right),
$$

assuming that the transmitter releases the $N_{A e m}$ information $A$ molecules at $t=0$. It is straightforward to take the derivative of 12 with respect to $t$ to find the time, $t_{\max }$, at which the maximum number of molecules is expected, found as

$$
t_{\text {max }}=\frac{-3+\sqrt{9+\left(4 k_{1} C_{E_{T o t}}\left|\vec{r}_{0}\right|^{2}\right) / D_{A}}}{4 k_{1} C_{E_{T o t}}},
$$

where we only consider non-negative finite time. The maximum number of expected molecules at the receiver, $\overline{N_{A_{\max }}}$, is then found by substituting (13) into $(12)$. By comparison, when there are no enzymes present, i.e., $C_{E_{T o t}}=0$, we substitute (4) instead of (10) into (11) and find that the maximum number of molecules is expected at time

$$
\left.t_{\text {max }}\right|_{C_{E_{T o t}}=0}=\frac{\left|\vec{r}_{0}\right|^{2}}{6 D_{A}},
$$

and it is straightforward to show that, for all valid (i.e., nonnegative) parameter values, $t_{\max } \leq\left. t_{\max }\right|_{C_{E_{T o t}}=0}$. So, when enzymes are added, the maximum number of molecules is expected no later than when enzymes are not added.

By inspection of 12 , we also observe that, for a given $t$, we expect to observe fewer $A$ molecules when enzymes are present, i.e., $\overline{N_{A o b}}(t) \leq\left.\overline{N_{A o b}}(t)\right|_{C_{E_{T o t}}=0}, \forall t$. Thus, we immediately have that $\overline{N_{A \max }} \leq\left.\overline{N_{A \max }}\right|_{C_{E_{T o t}}=0}$. In addition, when enzymes are present, the expected number of molecules will decrease to any value sooner than when enzymes are absent, i.e., ISI must decrease. In order to consider the selection of an appropriate bit interval $T_{B}$, we are interested in solving for the time required for $\overline{N_{A o b}}(t)$ to decrease to some threshold value, i.e., find $t_{\alpha}$ that satisfies

$$
\frac{\overline{N_{A o b}}\left(t_{\alpha}\right)}{\overline{N_{A \max }}} \leq \alpha,
$$

where $0<\alpha<1$ is a threshold fraction of the maximum expected number of molecules and $t_{\alpha}>t_{\max }$. Solving (15) presents two challenges. First, it cannot be strictly satisfied because we only have a lower bound on $\overline{N_{A o b}}(t)$ when enzymes are present; showing that the lower bound is lower than $\alpha \overline{N_{A \max }}$ does not satisfy $(15)$. However, we will assume that the system parameters are such that the lower bound (12) is met with equality; i.e., we assume that $k_{2} \rightarrow \infty$ and $k_{-1} \rightarrow 0$. Second, an analytical solution to 15 using the lower bound is not possible; further bounding will be required to obtain a closed-form expression for $t_{\alpha}$. Alternatively, we can solve (15) numerically using (12) without any further bounding by initializing $t_{\alpha}=t_{\max }$ and gradually increasing $t_{\alpha}$ by increments much smaller than $t_{\max }$ until the inequality in (15) is satisfied. The numerical solution enables us to assess the accuracy of a closed-form expression.

We derive a bound for $t_{\alpha}$ that satisfies (15) by first observing that, for smaller values of $t_{\alpha}$ (and over the range that we are interested in), the decay of $(12)$ is dominated by $t_{\alpha}{ }^{-\frac{3}{2}}$ and not the exponential. Therefore, we propose applying an upper bound on the exponential term by replacing it with its maximum value. It can be shown that the exponential term in 12 has maximum value $\exp \left(\left|\vec{r}_{0}\right| \sqrt{k_{1} C_{E_{T o t}} / D_{A}}\right)$ when $t_{\alpha}=\left|\vec{r}_{0}\right| / \sqrt{4 k_{1} C_{E_{T o t} D_{A}}}$. We replace the exponential term with this maximum, substitute (12) into (15), and solve for $t_{\alpha}$ as

$$
t_{\alpha} \geq \frac{1}{4 \pi D_{A}}\left(\frac{V_{o b} N_{A e m}}{\alpha \overline{N_{A \max }}}\right)^{\frac{2}{3}} \exp \left(-\frac{2}{3}\left|\vec{r}_{0}\right| \sqrt{\frac{k_{1} C_{E_{T o t}}}{D_{A}}}\right) .
$$

In addition, we can guarantee that $t_{\alpha}>t_{\max }$ because we used the upper-bound on the exponential term. Similarly, for the case without enzymes present, we replace the exponential term with its maximum value of 1 and find that

$$
\left.t_{\alpha}\right|_{C_{E_{T o t}=0}} \geq \frac{1}{4 \pi D_{A}}\left(\frac{V_{o b} N_{A e m}}{\left.\alpha \overline{N_{A \max }}\right|_{C_{E_{T o t}}=0}}\right)^{\frac{2}{3}}
$$

satisfies (15). A strict comparison between (16) and (17) is not fair due to the challenges previously mentioned. However, these expressions do provide some guidance in the selection of an appropriate bit interval. We will generally assume that the bit interval time $T_{B}$ is sufficiently long so that $T_{B}>t_{\max }$.

\section{B. Error Rate at the Receiver}

In our simple detection scheme, the receiver counts the number of free information $A$ molecules at time $t_{\max }$ after the 
start of the bit interval and compares that number with decision threshold $\xi$. We assume that there is perfect synchronization between the transmitter and receiver to emphasize the limitations of intersymbol interference. As noted at the beginning of this section, our proposed detector approximates a physically realizable detector; we do not expect to easily achieve perfect synchronization between devices. Recalling that $T_{B}$ is the bit interval time, the decision sampling time for the $j$ th bit interval is $(j-1) T_{B}+t_{\max }$.

Let $W[j]$ be the $j$ th information bit sent by the transmitter, i.e., sent at the beginning of the $j$ th bit interval, and let the $a$ priori probabilities of the transmitted bits be $\operatorname{Pr}(W[j]=1)=$ $P_{1}$ and $\operatorname{Pr}(W[j]=0)=P_{0}=1-P_{1}$, where $\operatorname{Pr}(\cdot)$ denotes probability. Let $\hat{W}[j]$ be the $j$ th received bit at the receiver. Thus, the reception mechanism can be written as

$$
\hat{W}[j]= \begin{cases}1 & \text { if } N_{A o b}\left((j-1) T_{B}+t_{\max }\right) \geq \xi \\ 0 & \text { if } N_{A o b}\left((j-1) T_{B}+t_{\max }\right)<\xi .\end{cases}
$$

It is clear that an error occurs if $W[j] \neq \hat{W}[j]$, and we define the error probability of the $j$ th bit $P_{e}[j]=$ $\operatorname{Pr}(W[j] \neq \hat{W}[j])$. So, we are interested in evaluating $\operatorname{Pr}\left(N_{A o b}\left((j-1) T_{B}+t_{\max }\right) \geq \xi\right)$, a function of the current and all previous emissions by the transmitter. We begin by considering the first bit, i.e., $j=1$, and then extend the result to any $j$ th bit in the transmission. Generally, bits transmitted later will have a higher probability of being detected in error because there are more previous bits to create ISI.

Consider the first bit for the case $W[1]=1$ (for the case $W[1]=0$ there are no information $A$ molecules anywhere in the system at the time $t_{\max }<T_{B}$ and so there will be none observed at the receiver). The lower bound on the expected number of observed molecules is also a lower bound on the probability density function (PDF) over all time and space for a single molecule if we set $N_{A e m}=1$ and assume that the location and state of any one $A$ molecule is independent of the other $A$ molecules. Therefore, a lower bound on the probability $P_{o b}(t)$ that a given molecule is observed within the receiver volume $V_{o b}$ at time $t$ is found by integrating (10) over $V_{o b}$. However, we recall from (11) that we simplified the integration by assuming that the concentration of molecules within the receiver is uniform and equal to that expected at the center of the receiver. Thus, we write

$$
P_{o b}(t) \geq \frac{V_{o b}}{\left(4 \pi D_{A} t\right)^{3 / 2}} \exp \left(-k_{1} C_{E_{T o t}} t-\frac{\left|\vec{r}_{0}\right|^{2}}{4 D_{A} t}\right),
$$

and we will assume that 19 is met with equality, i.e., we assume that $k_{2} \rightarrow \infty$ and $k_{-1} \rightarrow 0$. Generally, we have $N_{A \text { em }}$ information molecules, and each molecule is either inside $V_{o b}$ at a given time or outside, so the number of observed molecules follows the binomial distribution. Thus, we can write [34, Ch. 3]

$$
\begin{aligned}
& \operatorname{Pr}\left(N_{A_{o b}}(t) \geq \xi\right)= \\
& \sum_{w=\xi}^{N_{A_{e m}}}\left(\begin{array}{c}
N_{A e m} \\
w
\end{array}\right) P_{o b}(t)^{w}\left(1-P_{o b}(t)\right)^{N_{A e m}-w}
\end{aligned}
$$

Eq. 20 is exact for a given $P_{o b}(t)$ but is difficult to evaluate for large values of $N_{A e m}$. However, as noted in [10], we can write 20 in an equivalent form as

$$
\operatorname{Pr}\left(N_{A o b}(t) \geq \xi\right)=I_{P_{o b}}\left(\xi, N_{A e m}-\xi+1\right),
$$

where $I_{P_{o b}}(\cdot, \cdot)$ is the regularized incomplete beta function based on individual probability $P_{o b}(t)$, i.e., [35. Eq. 8.392]

$$
I_{P_{o b}}(a, b)=\frac{\int_{0}^{P_{o b}} t^{a-1}(1-t)^{b-1} d t}{\int_{0}^{1} t^{a-1}(1-t)^{b-1} d t} .
$$

Furthermore, we also note that

$$
\begin{aligned}
\operatorname{Pr}\left(N_{A o b}(t)=w\right)= & I_{P_{o b}}\left(w, N_{A e m}-w+1\right) \\
& -I_{P_{o b}}\left(w+1, N_{A e m}-w\right) .
\end{aligned}
$$

Eqs. (21) and 23 can be evaluated numerically but the incomplete beta function does not easily lend itself to optimization. For example, its derivative cannot be written in closed form. We consider two approximations of the binomial distribution. For infinitely large $N_{A e m}$ and infinitely small $P_{o b}(t)$, such that their product is a finite positive number, the binomial distribution approaches the Poisson distribution with mean $\overline{N_{A o b}}(t)=N_{A e m} P_{o b}(t)$, and we can write $[34$ Ch. 3]

$$
\left.\operatorname{Pr}\left(N_{A o b}(t)=w\right)\right|_{\text {Poiss }}=\frac{\overline{N_{A o b}}(t)^{w} \exp \left(-\overline{N_{A o b}}(t)\right)}{w !},
$$

and so

$\left.\operatorname{Pr}\left(N_{A o b}(t) \geq \xi\right)\right|_{\text {Poiss }}=1-\exp \left(-\overline{N_{A o b}}(t)\right) \sum_{w=0}^{\xi-1} \frac{\overline{N_{A o b}}(t)^{w}}{w !}$.

Alternatively, we can approximate the binomial distribution with a Gaussian distribution with mean $\overline{N_{A_{o b}}}(t)$ and variance $\overline{N_{A o b}}(t)\left(1-P_{o b}(t)\right)$. This approximation has been applied by other authors for molecular communication, cf. e.g. [11], [14], and is valid when $P_{o b}(t)$ is not close to one or zero and $\overline{N_{A o b}}(t)$ is sufficiently large. Generally, this approximation will not be as accurate as using the Poisson distribution because we will tend to have very small values for $P_{o b}(t)$. We still consider the Gaussian distribution because it does not include any factorials and so can be more computationally efficient than the Poisson distribution. The Gaussian approximation enables us to write

$$
\left.\operatorname{Pr}\left(N_{A o b}(t)=w\right)\right|_{\text {Gauss }}=\frac{\exp \left(\frac{\left(w-\overline{N_{A o b}}(t)\right)^{2}}{2 \overline{N_{A o b}}(t)\left(1-P_{o b}(t)\right)}\right)}{\sqrt{2 \pi \overline{N_{A o b}}(t)\left(1-P_{o b}(t)\right)}},
$$

and by using the error function [36, p. 406] we can show that

$$
\begin{aligned}
& \left.\operatorname{Pr}\left(N_{A o b}(t) \geq \xi\right)\right|_{\text {Gauss }} \\
& \quad=\frac{1}{2}\left[1-\operatorname{erf}\left(\frac{\xi-\overline{N_{A o b}}(t)}{\sqrt{2 \overline{N_{A o b}}(t)\left(1-P_{o b}(t)\right)}}\right)\right] .
\end{aligned}
$$


To evaluate the error probability for the first bit, we use either of 21), 25), or 27). As noted, an error in $\hat{W}[1]$ is possible only when $W[1]=1$. Thus, the probability of error in the first bit is

$$
P_{e}[1]=P_{1}\left[1-\operatorname{Pr}\left(N_{A o b}\left(t_{\max }\right) \geq \xi\right)\right] .
$$

The probability of error of the $j$ th bit is a function of all of the first $j$ bits, since information $A$ molecules can remain in the propagation environment from any of the previous emissions by the transmitter. The common practice when deriving error rates in the molecular communication literature is to assume that information $A$ molecules remain in the environment for no more than two transmission intervals, cf. e.g. [9]-[13]. We make no assumptions about how long information molecules remain in the proximity of the receiver in order to present a general bit error expression that includes all ISI, which has not yet been developed in the literature for a 3-dimensional environment. We will assume that the number of $A$ molecules, observed at some time $t$, that were emitted at the start of the $j$ th bit interval, are independent of the number of molecules, also observed at that time $t$, that were emitted at the start of any other bit interval. We note that this is not strictly true because an $E$ molecule that is bound to an $A$ molecule is temporarily unavailable to bind to the $A$ molecules of other transmissions (the mean time of unavailability is controlled by the value of degradation rate $k_{2}$, but we have assumed for analysis that $k_{2} \rightarrow \infty$ ).

From the independence of molecules emitted at the start of every bit interval, the number of molecules observed at the receiver at time $t$ is simply the sum of the number of molecules observed due to every emission. We emphasize that any molecule observed within the receiver volume $V_{o b}$ could have been emitted during the current or any previous bit interval. We define $N_{A o b}[j]=N_{A o b}\left((j-1) T_{B}+t_{\text {max }}\right)$, i.e., all information $A$ molecules observed at time $t_{\max }$ within the $j$ th bit interval, where $T_{B}$ is the bit interval time, and write

$$
N_{A o b}[j]=\sum_{i=1}^{j} N_{A o b}[j ; i],
$$

where $N_{A o b}[j ; i]$ is the number of molecules observed at the time $t_{\max }$ in the $j$ th bit interval that were emitted at the start of the $i$ th bit interval (we note that $N_{A_{o b}}[j ; i]=0, \forall j$ if $W[i]=0)$. Thus, $N_{A o b}[j]$ is a random variable that is a sum of random variables. From [37, Ch. 5], a random variable that is a sum of all Binomial, all Poisson, or all Gaussian random variables is also a Binomial, Poisson, or Gaussian random variable, respectively, and its mean is the sum of the means of the individual variables. We can then immediately write the expected number of molecules observed at the receiver at time $t_{\max }$ within the $j$ th bit interval as

$$
\overline{N_{A o b}}[j]=N_{A e m} \sum_{i=1}^{j} W[i] P_{o b}\left((j-i) T_{B}+t_{\max }\right),
$$

where $N_{\text {Aem }}$ is the number of molecules released when $W[i]=1$, and $P_{o b}(t)$ from $[19)$ is the probability that a single molecule is observed within $V_{o b}$ at time $t$ after its release from the transmitter. Given the mean $\overline{N_{A_{o b}}}[j], \operatorname{Pr}\left(N_{A_{o b}}[j]=w\right)$ can be evaluated from (24) using Poisson statistics or from 26 using Gaussian statistics, where $N_{A e m} P_{o b}(t)$ is replaced with $\overline{N_{A o b}}[j]$ (it is also possible to use Binomial statistics, but the adaptation of 23 is less straightforward so we omit the details here). Analogously, $\operatorname{Pr}\left(N_{A o b}[j] \geq \xi\right)$ can be evaluated from 25) using Poisson statistics or 27) using Gaussian statistics.

Given $\operatorname{Pr}\left(N_{A_{o b}}[j] \geq \xi\right)$, we can immediately evaluate $P_{e}[j]$ for a given transmitter bit sequence $W[i], i \in$ $\{1,2, \ldots, j\}$. Given the transmitter sequence for all prior intervals, i.e., $i<j$, the probability of error in the $j$ th bit is

$$
\begin{aligned}
P_{e}[j] & =P_{1} \operatorname{Pr}\left(N_{A o b}[j]<\xi \mid W[j]=1, W[i], i<j\right) \\
& +P_{0} \operatorname{Pr}\left(N_{A o b}[j] \geq \xi \mid W[j]=0, W[i], i<j\right) .
\end{aligned}
$$

If we have a priori knowledge of the current and all previous bits, then the expected probability of error is found by taking the appropriate term in (31). Generally, assuming no a priori knowledge, we must evaluate (31) for all $2^{j-1}$ possible prior bit sequences, though in practice we can average the expected error probability over a subset of all possible sequences.

The common practices in the literature of either ignoring ISI or only considering the interference caused by emission in the previous bit interval, cf. e.g. [9]-[11], [13], can both be evaluated as special cases of (31) by limiting the number of terms used in finding $\overline{N_{A o b}}[j]$ in 30 . Specifically, we initialize $i=j$ to ignore ISI and initialize $i=j-1$ to only consider ISI from the previous bit interval.

\section{Simulation FramewORK}

This section describes the framework used to perform stochastic simulations of the system of reaction-diffusion equations described by (6)-(8), which also simplifies to the diffusion-only case if the total enzyme concentration $C_{E_{T o t}}=$ 0 .

\section{A. Choice of Framework}

Our simulation framework uses a particle-based method, where the precise locations of all individual molecules are known. The primary alternative, subvolume-based methods, divide the environment into subvolumes and each molecule is known to be in a given subvolume. Particle-based methods tend to be less computationally efficient than subvolume-based methods, but they do not have to meet the latter's well-stirred requirement, where every subvolume should have many more nonreactive molecular collisions than reactive collisions, as described in [38], [39]. A general criterion for subvolume size is that the typical diffusion time for each species should be much less than the typical reaction time; see [40]. In order to satisfy this criterion, we would need to use very small subvolume sizes relative to the total size of the environment under consideration. If we used such small subvolumes, then we would not gain in computational efficiency and, for small (nanoscale) environments, the subvolume size would not be much greater than the size of individual molecules. Thus, we proceed with a particle-based method.

Every free molecule in a particle-based method diffuses independently along each dimension. Such methods require 
a constant global time step $\Delta t$ (the chosen value of $\Delta t$ represents a tradeoff in accuracy and simulation time) and there is a separation in the simulation of reaction and diffusion; see [41]. First, all free molecules are independently displaced along each dimension by generating normal random variables with variance $2 D_{S} \Delta t$, where $D_{S}$ is the diffusion coefficient of arbitrary species $S$. Next, potential reactions are evaluated to see whether they would have occurred during $\Delta t$. For bimolecular reactions, a binding radius $r_{B}$ is defined as how close the centers of two reactant molecules need to be at the end of $\Delta t$ in order to assume that the two molecules collided and bound during $\Delta t$. For unimolecular reactions, a random number is generated using the corresponding rate constant to declare whether the reaction occurred during $\Delta t$.

\section{B. Simulating Reactions}

All three reactions in (1) have an enzyme $E$ molecule as a reactant and an intermediate $E A$ molecule as a product (or vice versa). Thus, we must jointly consider the two unimolecular reactions with $E A$ as the reactant, and we must take care when modeling the binding and unbinding reactions so that the binding reaction does not occur when not intended.

The probability of the unbinding reaction $\left(k_{-1}\right)$ occuring is a function of both the unbinding and degradation rate constants, written as [41. Eq. 14]

$$
\operatorname{Pr}\left(\text { Reaction } k_{-1}\right)=\frac{k_{-1}}{k_{-1}+k_{2}}\left[1-\exp \left(-\Delta t\left(k_{-1}+k_{2}\right)\right)\right] \text {, }
$$

and the degradation reaction $\left(k_{2}\right)$ has an analogous expression by swapping $k_{-1}$ and $k_{2}$. A single random number uniformly distributed between 0 and 1 can then be used to determine whether a given $E A$ molecule reacts, and, if so, which reaction occurs.

The bimolecular binding reaction (i.e., the binding of an enzyme $E$ molecule and an information $A$ molecule at rate $k_{1}$ to form an intermediate $E A$ molecule) is reversible, so we must be careful in our choice of binding radius $r_{B}$, time step $\Delta t$, and what we assume when $E A$ reverts back to $E$ and $A$ molecules. If the $E$ and $A$ molecules are not physically separated when the unbinding reaction $\left(k_{-1}\right)$ occurs, and $r_{B}$ is much larger than the expected separation of the $E$ and $A$ molecules by diffusion in the following time step, then the binding reaction will very likely occur in the next time step regardless of the actual value of $r_{B}$. Therefore, we must consider the root mean square of the separation of $E$ and $A$ molecules in a single time step, given as [41, Eq. 23]

$$
r_{r m s}=\sqrt{2\left(D_{A}+D_{E}\right) \Delta t},
$$

where $D_{A}$ and $D_{E}$ are the constant diffusion coefficients of the $A$ and $E$ molecules, respectively. In general, an unbinding radius that is larger than $r_{B}$ is defined to separate the two molecules as soon as the reversible unbinding reaction occurs. The objective in doing so is to prevent the automatic rebinding of the same two molecules in the next time step and more accurately model the reaction kinetics; see [41]. However, if $r_{r m s} \gg r_{B}$, i.e., if the expected separation of the two molecules in one time step is much larger than the binding radius, then an unbinding radius is unnecessary and it is sufficient to keep the $A$ and $E$ molecules at the same coordinates when the unbinding reaction occurs. If $\Delta t$ is sufficiently large, then we can define $r_{B}$ as [41, Eq. 27]

$$
r_{B}=\left(\frac{3 k_{1} \Delta t}{4 \pi}\right)^{\frac{1}{3}}
$$

and this expression is only valid if $r_{r m s} \gg r_{B}$. Thus, if we are careful with our selection of $k_{1}$ and $\Delta t$, such that $r_{r m s} \gg r_{B}$ with $r_{B}$ given by (34), then we can legitimately use (34) to define $r_{B}$. If $r_{r m s} \gg r_{B}$ is not satisfied, then $r_{B}$ must be found using numerical methods; see [41]. In our simulations, we ensure that the use of (34) is justified.

When a pair of $A$ and $E$ molecules are within $r_{B}$ of each other, we move both of them to the midpoint of the line between their centers and re-label them as a single $E A$ molecule. If the corresponding unbinding reaction occurs in a later time step, then the molecule is re-labeled as separate $A$ and $E$ molecules and we do not change their locations until they diffuse in the following time step.

\section{Simulating the Transmitter and Receiver}

We simulate emissions at the transmitter by initializing $N_{A e m} A$ molecules centered at the origin and with a separation of $2 R_{A}$ between adjacent molecules so that together they form a spherical shape. The receiver can make observations only at integer multiples of the time step $\Delta t$, so for detection we round $t_{\max }$ to the nearest multiple of $\Delta t$. When an observation is made, all free $A$ molecules whose centers are within $V_{o b}$ are counted.

\section{Simulating an Unbounded Environment}

We noted in Section II that the physical environment is unbounded but the movement of enzyme $E$ molecules is restricted to the large volume $V_{\text {enz }}$. We force the enzymes to stay within $V_{e n z}$ by reflecting them off of the boundary of $V_{e n z}$ if diffusion carries them outside. In doing so, we simulate a uniform enzyme concentration using a finite number of molecules. However, we do not restrict the diffusion of information $A$ molecules. If an intermediate $E A$ molecule reaches the boundary of $V_{e n z}$, then we probabilistically decompose the molecule using the probabilities for the unbinding and degradation reactions as calculated by (32). As long as $V_{e n z}$ is sufficiently large, such that its boundary is far away from the receiver, then the impact of these forced reactions on the observations made at the receiver is negligible. In our simulations, we ensure that the side length of $V_{e n z}$ is at least three times greater than $\left|\vec{r}_{0}\right|$, i.e., the distance from the transmitter to the center of the receiver, and this is sufficient to ignore the behavior at the boundary of $V_{e n z}$ given the system parameter values that we use.

\section{E. Selecting Component Parameters}

Most enzymes are proteins and are usually on the order of less than $10 \mathrm{~nm}$ in diameter; see [4, Ch. 4]. From [2], smaller 
molecules diffuse faster, so we favor small molecules as information molecules. Many common small organic molecules, such as glucose, amino acids, and nucleotides, are about $1 \mathrm{~nm}$ in diameter. In the limit, single covalent bonds between two atoms are about $0.15 \mathrm{~nm}$ long; see [4, Ch. 2].

Higher rate constants imply faster reactions. Bimolecular rate constants can be no greater than the collision frequency between the two reactants, i.e., every collision results in a reaction. The largest possible value of $k_{1}$ is on the order of $1.66 \times 10^{-19}$ molecule ${ }^{-1} \mathrm{~m}^{3} \mathrm{~s}^{-1}$; see [27, Ch. 10] where the limiting rate is listed as on the order of $10^{8} \mathrm{~L} / \mathrm{mol} / \mathrm{s}$. $k_{-1}$ and $k_{2}$ usually vary between 1 and $10^{5} \mathrm{~s}^{-1}$, with values as high as $10^{7} \mathrm{~s}^{-1}$. In theory, we are not entirely limited to pre-existing enzyme-substrate pairs; protein and ribozyme engineering techniques can be used to modify and optimize the enzyme reaction rate, specificity, or thermal stability, or modify enzyme function in the presence of solvents; see [4. Ch. 10].

\section{Numerical And Simulation Results}

We present simulation results for an environment with a viscosity of $10^{-3} \mathrm{~kg} \cdot \mathrm{m}^{-1} \mathrm{~s}^{-1}$ and temperature of $25^{\circ} \mathrm{C}$. We compare three sets of system parameters, as described in Table I The chosen values of time step $\Delta t$ are selected large enough so that the root mean square separation between any two enzyme and information molecules in a single time step, $r_{r m s}$, is much greater than the binding radius, $r_{B}$ (we include the values of $r_{r m s}$ and $r_{B}$ calculated for each system in Table $\mathrm{I}$, and small enough so that a given information $A$ molecule is unlikely to enter and exit the receiver without being observed (the root mean square displacement of a single $A$ molecule in a single time step along one dimension, $\sqrt{2 D_{A} t}$, is always less than $r_{r m s}$, and $r_{r m s}$ is about half of the radius of the receiver). System 3 is identical to System 1 except for a larger value of $N_{A e m} . N_{E}$ is chosen so that the enzyme concentration in all systems is equivalent to $166 \mu \mathrm{M}$ (i.e., micromolar), which is high for a cellular enzyme; see [42]. In comparison to the limiting values of reaction rate constants discussed in the previous section, the reaction rate constants $k_{1}$ and $k_{2}$ for Systems 1 and 3 are relatively high due to the small size of the environments. The numbers of molecules $N_{A e m}$ and $N_{E}$ and the size of the environments are kept deliberately low in order to ease computation time.

\section{A. Accuracy of Expected Number of Molecules}

In Fig. 3, we compare the observed number of molecules for Systems 1 and 2 due to a single transmission. The observed number of $A$ molecules via simulation is averaged over at least 6000 independent emissions of $N_{\text {Aem }}$ molecules by the transmitter at $t=0$. We measure the number of information molecules observed over time, in comparison to the lower bound expression (12) and the expected number without enzymes in the environment as given by (12) for $C_{E_{T o t}}=0$.

We clearly see in Fig. 3 that the receivers in Systems 1 and 2 have the same lower bound on $\overline{N_{A o b}}(t)$, the expected number of observed information molecules, when we account for System 2's longer diffusion time as its receiver is placed
TABLE I

SYSTEM PARAMETERS USED FOR NUMERICAL AND SIMULATION RESULTS. THE VALUES FOR $r_{r m s}$ AND $r_{B}$ ARE CALCULATED FROM (33) AND 34, RESPECTIVELY.

\begin{tabular}{|c|c|c|c|}
\hline Parameter & System 1 & System 2 & System 3 \\
\hline$V_{\text {enz }}\left[\mu \mathrm{m}^{3}\right]$ & 1 & 37 & 1 \\
\hline$N_{\text {Aem }}$ & $5 \times 10^{3}$ & $5 \times 10^{3}$ & $2 \times 10^{4}$ \\
\hline$N_{E}$ & $10^{5}$ & $3.7 \times 10^{6}$ & $10^{5}$ \\
\hline$k_{1}\left[\frac{\mathrm{m}^{3}}{\text { molecule.s }^{3}}\right]$ & $2 \times 10^{-19}$ & $1.79 \times 10^{-20}$ & $2 \times 10^{-19}$ \\
\hline$k_{-1}\left[\mathrm{~s}^{-1}\right]$ & $10^{4}$ & 900 & $10^{4}$ \\
\hline$k_{2}\left[\mathrm{~s}^{-1}\right]$ & $10^{6}$ & $9 \times 10^{4}$ & $10^{6}$ \\
\hline$\left|\vec{r}_{0}\right|[\mathrm{nm}]$ & 300 & 1000 & 300 \\
\hline$\left|\vec{r}_{o b}\right|[\mathrm{nm}]$ & 45 & 150 & 45 \\
\hline$R_{A}[\mathrm{~nm}]$ & 0.5 & 0.5 & 0.5 \\
\hline$R_{E}[\mathrm{~nm}]$ & 2.5 & 2.5 & 2.5 \\
\hline$R_{E A}[\mathrm{~nm}]$ & 3 & 3 & 3 \\
\hline$\Delta t[\mu \mathrm{s}]$ & 0.5 & 5 & 0.5 \\
\hline$r_{r m s}[\mathrm{~nm}]$ & 22.9 & 72.4 & 22.9 \\
\hline$r_{B}[\mathrm{~nm}]$ & 2.88 & 2.77 & 2.88 \\
\hline
\end{tabular}
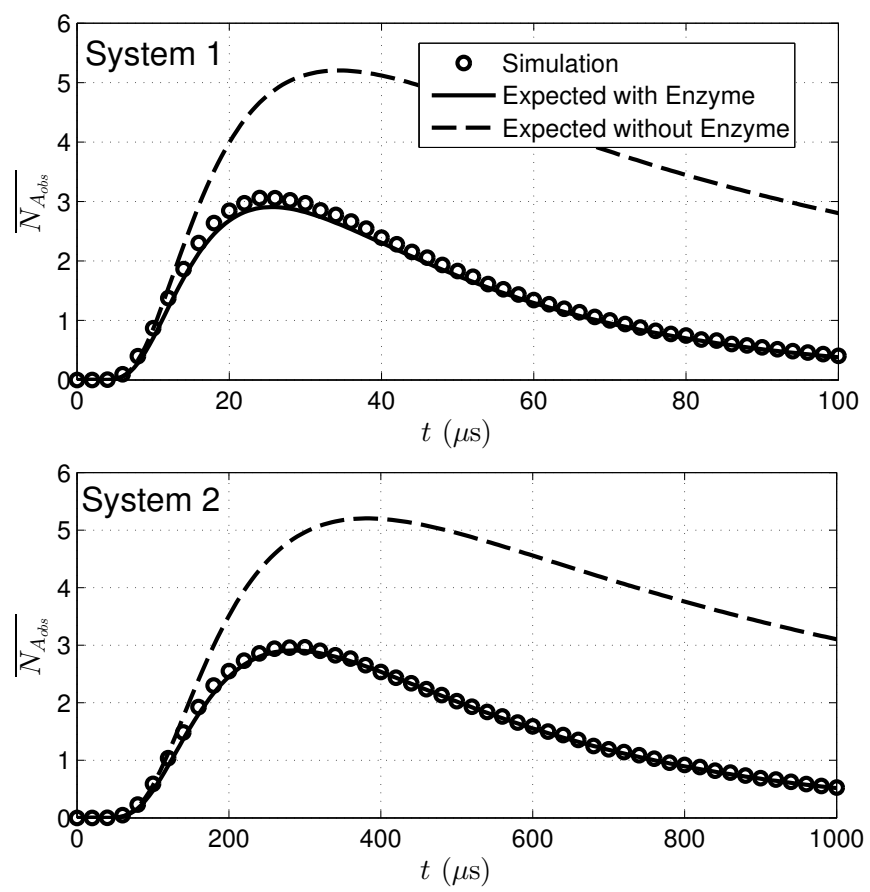

Fig. 3. Assessing the accuracy of the lower bound on the expected number of observed information molecules for Systems 1 (above) and 2 (below). The two systems have the same lower bound on the expected number of observed molecules when we account for System 2's longer diffusion time (the receiver is placed further away), but this bound is more accurate for System 2.

further away. The simulated number of observed molecules of both systems over time is close to the derived lower bound curve; the lower bound expression is accurate for describing the reduction in $\overline{N_{A o b}}(t)$ in comparison to not having enzymes present. However, the simulated value of $\overline{N_{A o b}}(t)$ for System 2 is visibly closer to the analytical lower bound expression 


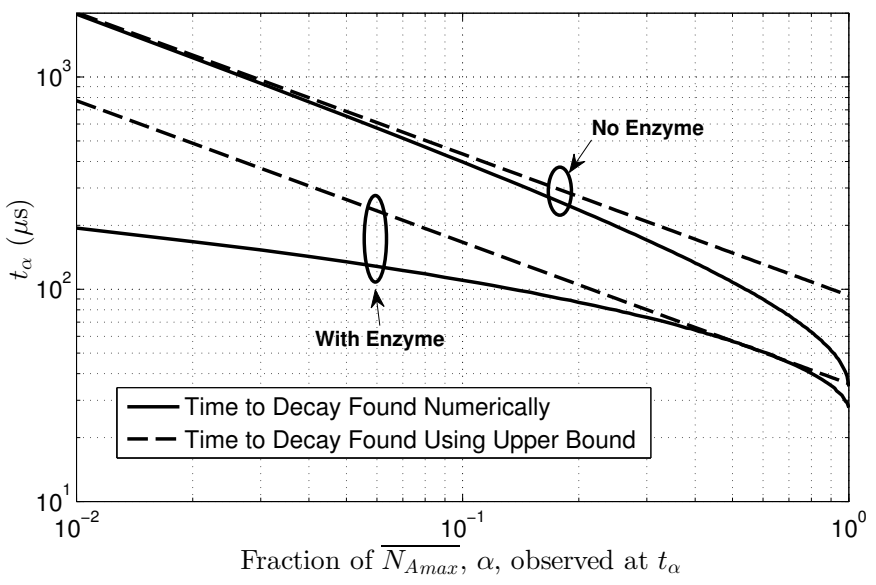

Fig. 4. Solving (15) for System 1 to determine how long it would take after a transmitter's single emission for the expected number of information molecules to decay to threshold fraction $\alpha$. The inequality is solved both numerically and by using upper bounds (16) and 17 for System 1 having enzymes present and absent, respectively.

than that for System 1. Further study of the effect of the environmental parameters (including chemical reactivity and the number of molecules) on the accuracy of the analytical lower bound expression can be found in [33].

For most of the remaining results, we focus on System 1 because it has a less accurate lower bound on the expected number of observed molecules and also because it has overall fewer molecules in the environment so that its simulations can be executed more efficiently.

\section{B. Selection of Bit Interval}

From (13) and (14) (or by observation of Fig. 3), we calculate that the maximum number of expected molecules for System 1 should be observed at times $t_{\max }=25.68 \mu \mathrm{s}$ and $\left.t_{\max }\right|_{C_{E_{T O t}}=0}=34.36 \mu \mathrm{s}$ with and without active enzymes, respectively. At these times, the expected number of observed molecules is $\overline{N_{A \max }}=2.92$ and $\left.\overline{N_{A \max }}\right|_{C_{E_{T o t}}=0}=5.20$, respectively. We are interested in solving (15) to get a sense of how long we should wait after an emission from the transmitter before sending another bit.

In Fig. 4 we solve 15 for the cases of enzymes present and absent by using upper bounds (16) and (17), respectively. We also solve (15) numerically. We see that the bound $(16)$, for enzymes present, is quite accurate if the fraction of molecules expected at the end of the interval is between $30 \%$ and $80 \%$ of the expected maximum (representing between 1 and 2.3 molecules expected on average), whereas the bound (17), for enzymes absent, improves with time as fewer molecules are expected.

Whether comparing the bounds or the numerical solutions, Fig. 4 shows that the transmitter can emit much more frequently with less risk of ISI if enzymes are present. For example, we may desire to have no more than $30 \%$ of $\overline{N_{A \max }}$ within the receiver at the end of the bit interval $T_{B}$. Using the numerical solution, we see that we would need to wait about $170 \mu \mathrm{s}$ if there were no enzymes present, but only about $70 \mu$ s with enzymes present. This result suggests that we

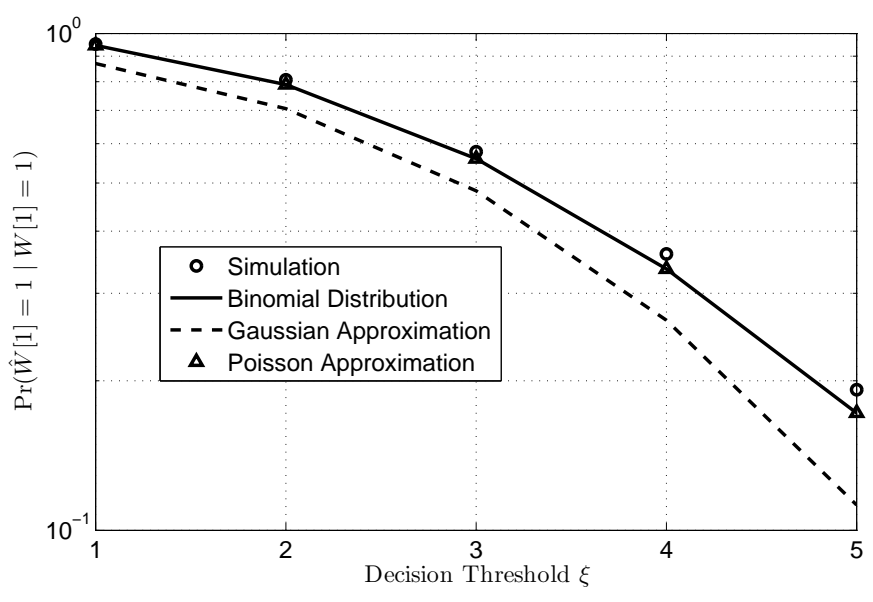

Fig. 5. Evaluating the detection probability for the first bit in System 1, i.e., $\operatorname{Pr}(\hat{W}[1]=1 \mid W[1]=1)$, as a function of decision threshold $\xi$.

can increase the data rate by about $150 \%$ with a comparable level of relative ISI. For lower levels of ISI, the numerical solutions suggest even higher increases in data transmission. We emphasize that solving (15) is insufficient in evaluating the ISI for a given set of system parameters, but it allows us to get a sense of what an appropriate $T_{B}$ might be.

\section{Detection Probability of One Bit Interval}

Before we consider the bit error rate over a lengthy data transmission, we consider the detection probability for the first emission by the transmitter. This enables us to focus on evaluating the accuracy of our expressions derived for the probability of the observed number of molecules being equal to or above some threshold; namely, we consider the binomial distribution (20), which is exact for a given $P_{o b}(t)$ (recall that we have (19), a lower bound on $P_{o b}(t)$ ), and the Poisson and Gaussian approximations (25) and (27), respectively. We evaluate the detection probabilities at time $t_{\max } \approx 25.5 \mu \mathrm{s}$ since we have to make observations at multiples of $\Delta t$ ( $\Delta t=0.5 \mu$ s for System 1), and we compare with the number of $A$ molecules observed via simulation as averaged over 6000 independent emissions by the transmitter at $t=0$. The results are presented in Fig. 5 for $1 \leq \xi \leq 5$.

In Fig. 5. we see that the detection probability can be kept above 0.5 for $\xi \leq 3$, which we expect since $\overline{N_{A \max }}=2.92$. The binomial distribution based on $P_{o b}(t)$ from 19 returns detection probabilities that are comparable to those found via simulation; for $\xi=2$, the detection probability found via simulation and via the binomial distribution are both about 0.8. We also see that the Poisson approximation is indistinguishable from the binomial distribution, whereas the Gaussian approximation has a notable loss in accuracy.

\section{Bit Error Rate of Multiple Intervals}

We now assess the bit error probability for System 1 transmitting multiple bits by comparing the evaluation of 31 with simulation results. We choose the Poisson approximation for evaluating the expected $P_{e}[j]$ because of its high accuracy to the binomial distribution for System 1. We also select either 


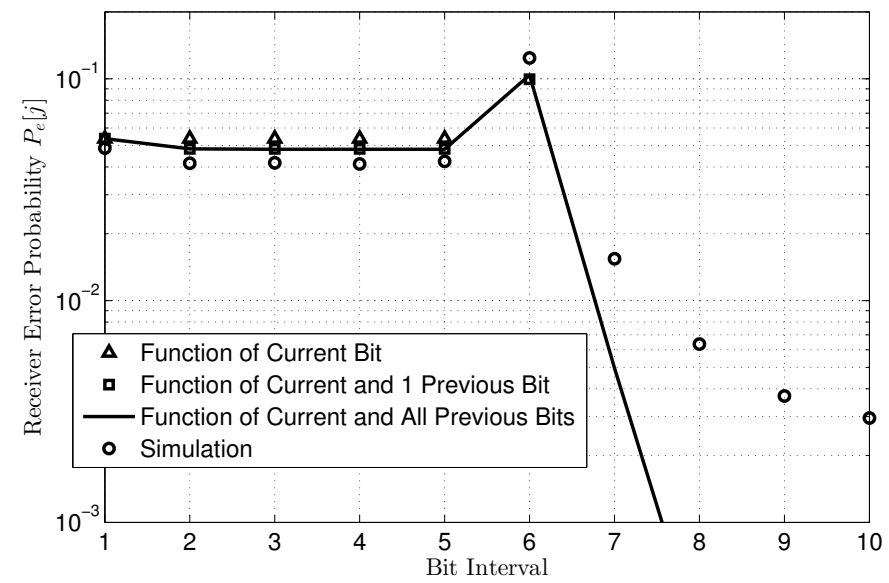

Fig. 6. Evaluating the error probability of System 1 over time with bit interval $T_{B}=120 \mu$ s and a known transmission sequence; five 1 s followed by five 0 s.

$T_{B}=50 \mu \mathrm{s}$ or $T_{B}=120 \mu \mathrm{s}$. For $T_{B}=120 \mu \mathrm{s}$, we see from Fig. 4 that the expected number of molecules at the end of a bit interval due to a single emission of molecules is less than $20 \%$ of the maximum when enzymes are present but more than $40 \%$ of the maximum when there are no enzymes. For $T_{B}=50 \mu \mathrm{s}$, the expected ISI is even higher.

First, we consider a known data sequence in order to compare the accuracy of missed detection (incorrectly detecting a 1 instead of a 0 ) versus false alarm (incorrectly detecting a 0 instead of a 1). The transmitter emits molecules according to a sequence of five consecutive 1 s followed by five consecutive 0s. In Fig. 6, we track the receiver error probability $P_{e}[j]$ over time for $T_{B}=120 \mu \mathrm{s}$, where the simulation results are averaged over 35000 independent transmissions. Receiver errors within the first five bit intervals are missed detections, whereas errors within the last five bit intervals are false alarms. We evaluate the error probability using only knowledge of the current bit, using knowledge of the current and previous bits, and using knowledge of the current and all previous bits (up to nine). We set decision threshold $\xi=1$ as we will later see that it is the optimal threshold for System 1 when $T_{B}=120 \mu \mathrm{s}$.

In Fig. 6, we see via both simulation and evaluation of 31 that the error probability reaches a floor on missed detection with repeated $1 \mathrm{~s}$ and tends to zero on false alarm with repeated $0 \mathrm{~s}$, which is an intuitive result. Note that, when the transmitted bit changes at interval 6 , the error probability assuming no ISI immediately drops to zero whereas all other curves spike sharply upwards, showing a high probability of false alarm when a 0 is transmitted after a 1 . The error probability using knowledge of only the current and previous bits drops to zero by interval 7 , even though the error measured via simulation and evaluated by considering the current and all previous bits shows a non-negligible error probability of about $1 \%$. All evaluations of (31) appear to over-estimate missed detection and under-estimate false alarm; this makes sense since the underlying probability of observing an information molecule is a lower bound. Thus, the accuracy of the expected error probability, even when considering all previous bits, becomes quite poor when consecutive zeros are transmitted. However,

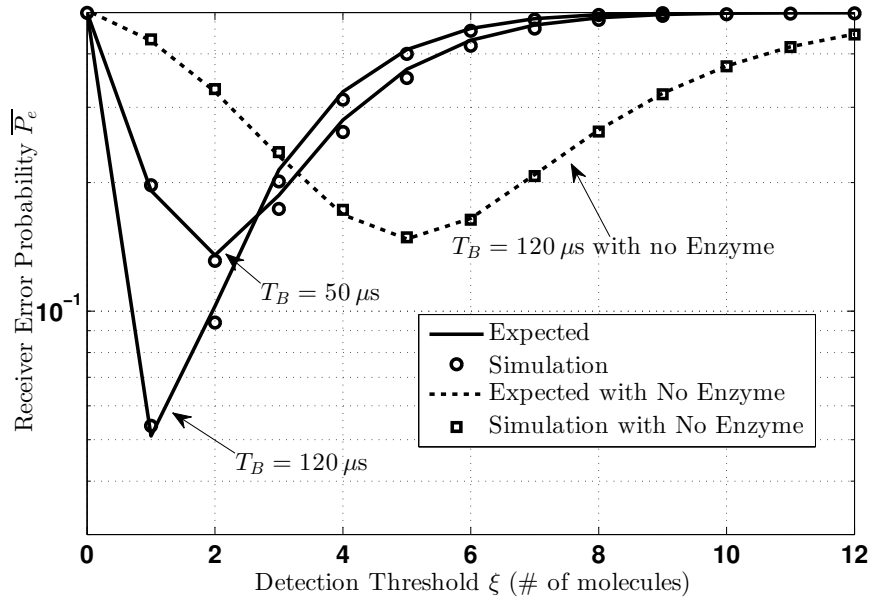

Fig. 7. Evaluating the error probability of System 1 as a function of the bit decision threshold $\xi$ at the receiver for bit interval $T_{B}=50 \mu$ s and $T_{B}=$ $120 \mu$ s with enzymes and $T_{B}=120 \mu$ s without enzymes. The transmission is a sequence of 50 randomly generated bits.

we will next see that this does not have a noticeable effect on the average bit error probability for a random transmission in System 1.

We now assess the mean receiver error probability, $\bar{P}_{e}$, as a function of the bit decision threshold where we generate a long random source transmission (50 bits). We assume no a priori knowledge of the transmitted data when calculating the expected error probability $P_{e}[j]$ from $[31$, where all prior bit intervals are considered and we average $P_{e}[j]$ over 1000 random bit sequences, and $\bar{P}_{e}$ is evaluated by averaging $P_{e}[j]$ over all $j$. The results are presented in Fig. 7 for $T_{B}=50 \mu \mathrm{s}$ and $T_{B}=120 \mu \mathrm{s}$ where we set the a priori bit probabilities $P_{1}=P_{0}=0.5$. We also consider $T_{B}=120 \mu$ s when there are no enzymes present. Simulation results are averaged over 6000 independent transmissions.

We see that in Fig. 7 the optimal decision threshold for System 1 and $T_{B}=120 \mu \mathrm{s}$ is $\xi=1$ with enzymes and $\xi=5$ without enzymes, whereas the optimal threshold when $T_{B}=50 \mu \mathrm{s}$ is $\xi=2$. These differences make intuitive sense; when the bit interval is shorter or enzymes are not present, there is more ISI from previous bits such that a lower decision threshold can result in many more false alarms. The minimum error probability is much lower for $T_{B}=120 \mu \mathrm{s}$ with enzymes; just over 0.05 versus over 0.12 for $T_{B}=120 \mu \mathrm{s}$ without enzymes and for $T_{B}=50 \mu \mathrm{s}$.

The error expected by the evaluation of 31) with enzymes is much more accurate than what we might expect from Fig. 6 alone; a long sequence of consecutive zeros is unlikely in a random transmission, and the slight over-estimation of missed detection is on averge balanced by the under-estimation of false alarm. The primary observation in Fig. 7 is that, by adding enzymes, the data transmission rate can be significantly increased (more than doubled here) while maintaining the same expected error probability, or the bit error probability can be significantly improved for the same data transmission rate.

Finally, we note that the error probabilities presented in 


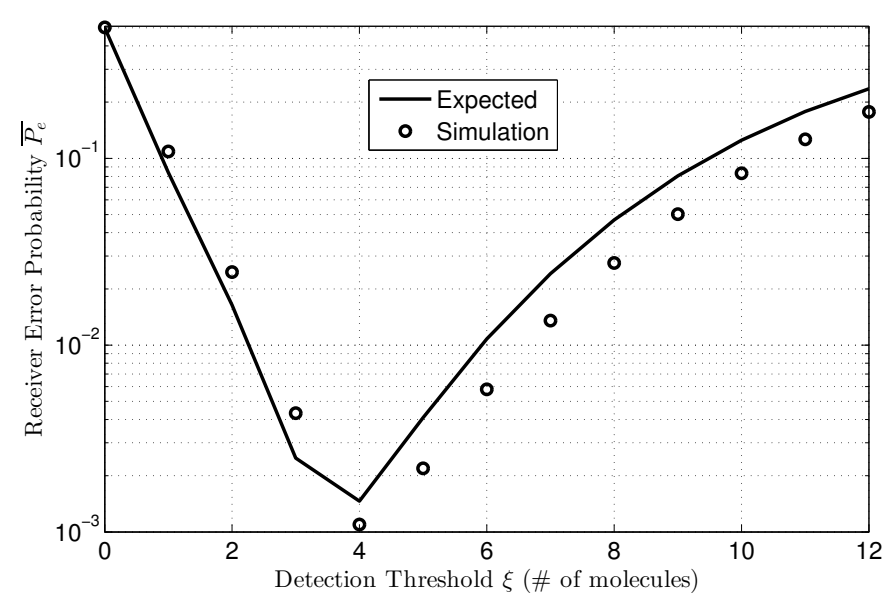

Fig. 8. Evaluating the error probability of System 3 as a function of the bit decision threshold $\xi$ at the receiver for bit interval $T_{B}=120 \mu \mathrm{s}$.

this section are not very low in the context of information transmission. By deliberately selecting a system with a low number of molecules, we were limited by a low expected maximum number of information molecules $\overline{N_{A \max }}$. For contrast, we consider System 3, for which we can expect $\overline{N_{A \max }}=11.69$ molecules to be observed at $t_{\max }=25.68 \mu \mathrm{s}$ when there is a single emission. We evaluate the average error probability $\bar{P}_{e}$ when the transmitter in System 3 emits a stream of 50 bits with $T_{B}=120 \mu \mathrm{s}$. The results are plotted in Fig. 8 . where we see that for the optimal threshold $\xi=4$, the expected error probability is about $1.5 \times 10^{-3}$, much less than those observed for System 1, and the observed error probability is about $10^{-3}$. The larger deviation between expected and simulated results for System 3 relative to System 1 is because the lower bound expression on the PDF (19) is not as tight for System 3; the over-estimation of missed detection and the under-estimation of false alarm are more evident for System 3 than they are for System 1 as shown in Fig. 7 .

\section{CONCLUSIONS AND Future WORK}

In this paper, we expanded upon the physical system model that we developed in [1] for the transmission of impulses of molecules being released into a propagation environment that contains diffusing enzymes. We derived a lower bound expression on the expected number of information molecules at the receiver. We showed how the expected signal degradation can be used to predict an appropriate bit interval length. We then derived the expected error rate for a simple receiver scheme as a function of the current and all previous emissions. Our results showed that the expected probability of error can be accurately represented by the Poisson approximation and agrees with the error probabilities observed via simulation. The presence of enzymes was shown to enable a decrease in the probability of error or an increase in the data transmission rate.

Our on-going work includes considering the impact of flow, external noise sources, and multiuser interference on a diffusive communication link. We are studying the design of practical diffusive detectors and comparing their performance to that of the optimal detector. We are also formalizing tractable optimization problems to minimize the probability of error when selecting the bit decision threshold and other transmission parameters.

\section{REFERENCES}

[1] A. Noel, K. C. Cheung, and R. Schober, "Improving diffusion-based molecular communication with unanchored enzymes," in Proc. 2012 ICST BIONETICS, Dec. 2012. [Online]. Available: arXiv:1305.1783

[2] I. F. Akyildiz, F. Brunetti, and C. Blazquez, "Nanonetworks: A new communication paradigm," Computer Networks, vol. 52, no. 12, pp. 2260-2279, May 2008.

[3] T. Nakano, M. J. Moore, F. Wei, A. V. Vasilakos, and J. Shuai, "Molecular communication and networking: Opportunities and challenges," IEEE Trans. Nanobiosci., vol. 11, no. 2, pp. 135-148, Jun. 2012.

[4] B. Alberts, D. Bray, K. Hopkin, A. Johnson, J. Lewis, M. Raff, K. Roberts, and P. Walter, Essential Cell Biology, 3rd ed. Garland Science, 2010.

[5] S. Kadloor and R. Adve, "A framework to study the molecular communication system," in Proc. 2009 IEEE ICCCN, Aug. 2009, pp. 1-6.

[6] A. Einolghozati, M. Sardari, A. Beirami, and F. Fekri, "Capacity of discrete molecular diffusion channels," in Proc. 2011 IEEE ISIT, Aug. 2011, pp. 723-727.

[7] S. Kadloor, R. R. Adve, and A. W. Eckford, "Molecular communication using Brownian motion with drift," IEEE Trans. Nanobiosci., vol. 11, no. 2, pp. 89-99, Jun. 2012.

[8] K. V. Srinivas, A. W. Eckford, and R. S. Adve, "Molecular communication in fluid media: The additive inverse Gaussian noise channel," IEEE Trans. Inf. Theory, vol. 58, no. 7, pp. 4678-4692, Jul. 2012.

[9] T. Nakano, Y. Okaie, and A. V. Vasilakos, "Throughput and efficiency of molecular communication between nanomachines," in Proc. 2012 IEEE WCNC, Apr. 2012, pp. 704-708.

[10] M. S. Kuran, H. B. Yilmaz, T. Tugcu, and B. Ozerman, "Energy model for communication via diffusion in nanonetworks," Nano Commun. Net., vol. 1, no. 2, pp. 86-95, Jun 2010.

[11] M. S. Kuran, H. B. Yilmaz, T. Tugcu, and I. F. Akyildiz, "Modulation techniques for communication via diffusion in nanonetworks," in Proc. 2011 IEEE ICC, Jun. 2011, pp. 1-5.

[12] M. U. Mahfuz, D. Makrakis, and H. T. Mouftah, "A comprehensive study of concentration-encoded unicast molecular communication with binary pulse transmission," in Proc. 2011 IEEE NANO, Aug. 2011, pp. 227-232.

[13] B. Atakan, S. Galmes, and O. B. Akan, "Nanoscale communication with molecular arrays in nanonetworks," IEEE Trans. Nanobiosci., vol. 11, no. 2, pp. 149-160, Jun. 2012.

[14] B. Atakan and O. B. Akan, "Deterministic capacity of information flow in molecular nanonetworks," Nano Commun. Net., vol. 1, no. 1, pp. 31-42, Mar. 2010.

[15] D. Miorandi, "A stochastic model for molecular communications," Nano Commun. Net., vol. 2, no. 4, pp. 205-212, Dec. 2011.

[16] M. J. Moore, T. Suda, and K. Oiwa, "Molecular communication: Modeling noise effects on information rate," IEEE Trans. Nanobiosci., vol. 8, no. 2, pp. 169-180, Jun. 2009.

[17] L.-S. Meng, P.-C. Yeh, K.-C. Chen, and I. F. Akyildiz, "MIMO communications based on molecular diffusion," in Proc. 2012 IEEE GLOBECOM, Dec. 2012, pp. 5602-5607.

[18] M. S. Leeson and M. D. Higgins, "Forward error correction for molecular communications," Nano Commun. Net., vol. 3, no. 3, pp. 161-167, Sep. 2012.

[19] M. Pierobon and I. F. Akyildiz, "Intersymbol and co-channel interference in diffusion-based molecular communication," in Proc. 2012 IEEE ICC MONACOM, Jun. 2012, pp. 6126-6131.

[20] L.-S. Meng, P.-C. Yeh, K.-C. Chen, and I. F. Akyildiz, "Optimal detection for diffusion-based communications in the presence of ISI," in Proc. 2012 IEEE GLOBECOM, Dec. 2012, pp. 3843-3848.

[21] P.-J. Shih, C.-H. Lee, and P.-C. Yeh, "Channel codes for mitigating intersymbol interference in diffusion-based molecular communications," in Proc. 2012 IEEE GLOBECOM, Dec. 2012, pp. 4444-4448.

[22] H. ShahMohammadian, G. G. Messier, and S. Magierowski, "Optimum receiver for molecule shift keying modulation in diffusion-based molecular communication channels," Nano Commun. Net., vol. 3, no. 3, pp. 183-195, Sep. 2012.

[23] M. Pierobon and I. F. Akyildiz, "Capacity of a diffusion-based molecular communication system with channel memory and molecular noise," IEEE Trans. Inf. Theory, vol. 59, no. 2, pp. 942-954, Feb. 2013. 
[24] P. Nelson, Biological Physics: Energy, Information, Life, updated 1st ed. W. H. Freeman and Company, 2008.

[25] C. T. Chou, "Extended master equation models for molecular communication networks," IEEE Trans. Nanobiosci,, vol. 12, no. 2, pp. 79-92, Jun. 2013.

[26] M. S. Kuran, H. B. Yilmaz, and T. Tugcu, "A tunnel-based approach for signal shaping in molecular communication," in Proc. 2013 IEEE ICC MONACOM, Jun. 2013, pp. 1-6.

[27] R. Chang, Physical Chemistry for the Biosciences. University Science Books, 2005.

[28] I. Llatser, E. Alarcon, and M. Pierobon, "Diffusion-based channel characterization in molecular nanonetworks," in Proc. 2011 IEEE INFOCOM, Apr. 2011, pp. 467-472.

[29] M. J. Moore, T. Nakano, A. Enomoto, and T. Suda, "Measuring distance from single spike feedback signals in molecular communication," IEEE Trans. Signal Process., vol. 60, no. 7, pp. 3576-3587, Jul. 2012.

[30] M. Pierobon and I. F. Akyildiz, "Information capacity of diffusionbased molecular communication in nanonetworks," in Proc. 2011 IEEE INFOCOM 2011, Apr. 2011, pp. 506-510.

[31] M. U. Mahfuz, D. Makrakis, and H. T. Mouftah, "Characterization of intersymbol interference in concentration-encoded unicast molecular communication," in Proc. 2011 IEEE CCECE, May 2011, pp. 164-168.

[32] L. Debnath, Nonlinear Partial Differential Equations for Scientists and Engineers, 2nd ed. Birkhaeuser, 2005.

[33] A. Noel, K. C. Cheung, and R. Schober, "Using dimensional analysis to assess scalability and accuracy in molecular communication," in Proc. 2013 IEEE ICC MONACOM, Jun. 2013, pp. 818-823. [Online]. Available: dx.doi.org/10.1109/ICCW.2013.6649346

[34] M. Ullah and O. Wolkenhauer, Stochastic Approaches for Systems Biology. Springer, 2011.

[35] I. S. Gradshteyn and I. M. Ryzhik, Table of Integrals, Series, and Products, 5th ed. London: Academic Press, 1994.

[36] K. B. Oldham, J. C. Myland, and J. Spanier, An Atlas of Functions with Equator, the Atlas Function Calculator, 2nd ed. Springer, 2008.

[37] S. Ross, Introduction to Probability and Statistics for Engineers and Scientists, 4th ed. Academic Press, 2009.

[38] D. T. Gillespie, "Stochastic simulation of chemical kinetics," Annu. Rev. Phys. Chem., vol. 58, no. 1, pp. 35-55, May 2007.

[39] K. A. Iyengar, L. A. Harris, and P. Clancy, "Accurate implementation of leaping in space: the spatial partitioned-leaping algorithm," J. Chem. Phys., vol. 132, no. 9, p. 094101, Mar. 2010.

[40] D. Bernstein, "Simulating mesoscopic reaction-diffusion systems using the Gillespie algorithm," Phys. Rev. E, vol. 71, no. 4, p. 041103, Apr. 2005.

[41] S. S. Andrews and D. Bray, "Stochastic simulation of chemical reactions with spatial resolution and single molecule detail," Physical Biology, vol. 1, no. 3, p. 137, Aug. 2004.

[42] K. R. Albe, M. H. Butler, and B. E. Wright, "Cellular concentrations of enzymes and their substrates," J. Theor. Biol., vol. 143, no. 2, pp. 163-195, Mar. 1990.

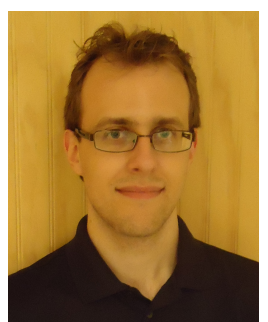

Adam Noel (S'09) received the B.Eng. degree from Memorial University in 2009 and the M.A.Sc. degree from the University of British Columbia (UBC) in 2011 , both in electrical engineering. He is now a $\mathrm{Ph} . \mathrm{D}$. candidate in electrical engineering at UBC, and in 2013 was a visiting researcher at the Institute for Digital Communications, Friedrich-AlexanderUniversität Erlangen-Nürnberg. His research interests include wireless communications and how traditional communication theory applies to molecular communication

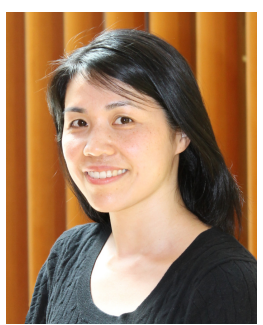

Karen C. Cheung received the B.S. and Ph.D. degrees in bioengineering from the University of California, Berkeley, in 1998 and 2002, respectively. From 2002 to 2005, she was a postdoctoral researcher at the Ecole Polytechnique Fdrale de Lausanne, Lausanne, Switzerland. She is now at the University of British Columbia, Vancouver, BC, Canada. Her research interests include lab-on-a-chip systems for cell culture and characterization, inkjet printing for tissue engineering, and implantable neural interfaces.

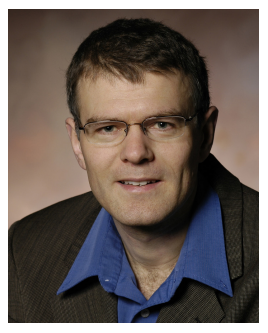

Robert Schober (S'98, M'01, SM'08, F'10) received the Diplom (Univ.) and the Ph.D. degrees in electrical engineering from the University of Erlangen-Nuremberg in 1997 and 2000, respectively. Since May 2002 he has been with the University of British Columbia (UBC), Vancouver, Canada, where he is now a Full Professor. Since January 2012 he is an Alexander von Humboldt Professor and the Chair for Digital Communication at the Friedrich Alexander University (FAU), Erlangen, Germany. His research interests fall into the broad areas of Communication Theory, Wireless Communications, and Statistical Signal Processing. He is currently the Editor-in-Chief of the IEEE Transactions on Communications. 